\title{
Prevalencia, severidad de caries dental y necesidad de tratamiento restaurador en escolares de 6 a 12 Años de la Provincia de Santiago, Región Metropolitana.
}

\section{Prevalence, severity of dental caries, and treatments needs, among 6 to 12 years old school children, in Santiago-Metropolitan Region.}

\author{
M.Consuelo Fresno ${ }^{1 *}$, Gabriela Jeldes ${ }^{2}$,Juan Estay ${ }^{1}$, Javier Martin $^{1}$.
}

\author{
1. Dpto. Odontología Restauradora . Facultad de \\ Odontología, Universidad de Chile. Chile \\ 2. Centro Comunitario de Salud Familiar (Cecosf) \\ Ayacara - Chaitén. Chile \\ * Correspondencia Autor: M.Consuelo Fresno | \\ Dirección: Olivos 943 | Teléfono: +569 84485601 \\ E-mail: mcfresno@odontologia.uchile.cl \\ Trabajo recibido el 15/03/2019. \\ Aprobado para su publicación el 20/05/2019
}

\begin{abstract}
RESUMEN
Objetivo: el objetivo de este estudio epidemiológico, fue determinar la prevalencia y severidad de caries dental, expresados en el índice de dientes definitivos con caries, obturados y perdidos (COPD), índice de dientes primarios con caries, extraídos y obturados (ceod) e Índice Significante de Caries (ISC) además establecer la necesidad de tratamiento restaurador y recursos humanos necesarios para ello, en una población escolar de 6 a 12 años de la Provincia de Santiago, Región Metropolitana. Metodología: Este estudio de corte transversal se realizó en 7 colegios entre públicos y privados que se encontraban debidamente registrados en la base de datos de la Secretaría Regional Ministerial de Educación pertenecientes a Provincia de Santiago, Región Metropolitana. Se seleccionó mediante un muestreo aleatorio estratificado por nivel socioeconómico, sexo y edad, una muestra de 1333 escolares de entre 6 a 12 años los cuales fueron evaluados previo asentimiento y con el consentimiento de sus tutores legales, por dos dentistas calibrados (Kappa 0.94). Para el diagnóstico de caries se utilizaron los criterios OMS. Los datos fueron recolectados en una ficha diseñada especialmente para ello y analizados utilizando el programa estadístico SPSS realizando pruebas no paramétricas de Kruskal-Wallis y U Mann-Whitney con un valor de significancia estadística del $95 \%$ $(p<0,05)$. Resultados La prevalencia de caries encontrada en esta población, en dentición permanente fue de $24,5 \%$, mientras que para dentición primaria $47,8 \%$, los valores obtenidos del Índice COPD 0,48; ceod 1,67; ISC para COPD 1,45 y ISC para ceod 4,52. Las horas profesionales de odontólogos/as con sus respectivos/ as asistentes, necesarias destinadas sólo a tratamiento restaurador para dar solución al daño encontrado en la muestra de estudio al momento del examen, corresponden a 167 horas para dentición permanente y 689 horas para dentición primaria. Conclusiones: La prevalencia y distribución de caries dental en escolares de 6 a 12 años de la Provincia de Santiago correspondió a cifras menores a las consignadas en el Diagnóstico de Salud Oral del Ministerio de Salud del año 2007, y menores a los índices propuestos por las organizaciones internacionales. Para resolver la necesidad de tratamiento restaurador en esta muestra se necesitarán 856 horas odontológicas.
\end{abstract}

PALABRAS CLAVE

Caries dental; Prevalencia; Severidad; Escolares; Necesidad de tratamiento.

Rev. Clin. Periodoncia Implantol. Rehabil. Oral Vol. 12(2); 81-86, 2019.

\section{ABSTRACT}

Objective: The aim of the study was to determine prevalence and severity of dental caries, treatment needs and human resources required, in a school population aged 6 to 12 years in province of Santiago. Methodology: This cross-sectional study was conducted in seven schools. A stratified random sample was selected by socioeconomic level, sex and age. 1333 school children were evaluated by two calibrated dentists (Kappa 0.94). WHO criteria were used for caries diagnosis. Data were statistically analyzed by nonparametric tests of Kruskal-Wallis and U MannWhitney, statistical significance value of $95 \%(p<0.05)$. Results: 1333 patients fit the inclusion criteria. Caries prevalence was $24.5 \%$ for permanent teeth and $47.8 \%$ for primary teeth. DMFT index was 0.48 , dmft index was 1.67 . SiC was 1.45 for DMFT and 4.52 for dmft. A total of 167 and 689 hours of restorative treatment were 
required for permanent dentition and primary dentition, respectively. Conclusions: The prevalence and severity distribution of dental caries in school children aged 6 to 12 years in the Province of Santiago were lower than those recorded in the past, and lower than the rates proposed by the international organizations. To restore damaged founded in this sample 856 hours will be needed.

\section{KEY WORDS}

Caries; Prevalence; Severity; School children, Treatment needs.

Rev. Clin. Periodoncia Implantol. Rehabil. Oral Vol. 12(2); 81-86, 2019.

\section{INTRODUCCIÓN}

La caries dental es la enfermedad crónica no transmisible más prevalente en Chile y el mundo, afectando tanto dentición primaria como permanente, representando un importante desafío para la salud pública ${ }^{(1,2)}$ La Organización Mundial de la Salud (OMS) ha estimado que entre el 60 y $90 \%$ de los niños a nivel global presentan lesiones de caries cavitadas, afectando a más de la mitad de la población en los países industrializados y, por ser un proceso acumulativo, va incrementándose la severidad del daño a medida que aumenta la edad ${ }^{(3,4)}$.

Desde la perspectiva de causalidad, las enfermedades que afectan a la cavidad oral como la caries, son enfermedades complejas ya que están originadas por la interacción de diferentes factores, y para su análisis se deben considerar la interacción de ellos tales como los componentes genéticos, epigenéticos, ambientales, culturales, sociales y locales $(5,6,7,8,9)$. La etiología heterogénea de estas enfermedades explica la dificultad de lograr su control y tiene un impacto directo no solo sobre la salud oral del individuo sino también sobre su salud general(10).

Como consecuencias de la caries dental se puede mencionar dolor, abscesos locales, infección sistémica y otras condiciones graves de salud que, y si se considera que es una de las enfermedades infantiles crónicas más comunes, pueden conducir a la ausencia escolar, actividad restringida, necesidad de atención de urgencia, hospitalización, capacidad disminuida para aprender o incluso insuficiencia física de desarrollo especialmente la altura y peso del niño. Así mismo, el dolor dental influye directamente en desmedro de la calidad de vida de los individuos ${ }^{(11)}$.

El fortalecimiento en el enfoque social de los determinantes de salud ha dado mayor importancia al entorno social, tanto en la determinación del estado de salud oral de la población como en la respuesta en términos de intervenciones sanitarias,en lugar de enfocarse sólo en cambios del comportamiento individual(12).

La información sobre el estado de salud oral es esencial para la toma de decisiones a nivel gubernamental que involucren la prevención y tratamiento de éstas, por ello es importante el levantamiento de evidencia para poder trazar una línea base sobre la condición actual de salud oral en nuestro país y de esta forma poder comparar en el tiempo si existe modificación de estas cifras, como así también poder evaluar indirectamente el impacto de las distintas medidas llevadas a cabo y fortalecer la implementación de políticas públicas para mejorar la salud oral de los grupos objetivos.

En Chile, los estudios realizados por el Ministerio de Salud en el año 2007 demostraron la presencia de altos índices de caries en la población infantil, con una prevalencia de $70,3 \%$ en escolares de 6 años y de $62,5 \%$ en los de $12^{(13,14)}$

El presente estudio tiene como objetivo determinar la prevalencia y severidad de caries y necesidad de tratamiento de una población representativa de escolares de 6 a 12 años de la Provincia de Santiago.

\section{MATERIAL Y MÉTODO}

\section{Diseño y muestra del estudio}

La muestra de este estudio representa al universo de escolares de la Región Metropolitana del período comprendido entre 2015 y 2017. Se incluyeron siete colegios, uno del estrato socioeconómico alto, representando el $20,78 \%$ de la muestra, cinco del estrado medio siendo el $53,71 \%$ y uno del estrato bajo con el $25,50 \%$ de la muestra. Estos establecimientos no contaban con intervenciones preventivas adicionales a los entregados en los programas odontológicos regulares del Ministerio de Salud (MINSAL). Para esto, las 32 comunas de la Provincia de Santiago se estratificaron socioeconómicamente considerando la Encuesta CASEN, del año 2013, en estrato I alto, II medio y III bajo. Dentro de cada estrato se eligieron en forma aleatoria las comunas, luego los establecimientos educacionales entre aquellos que se encontraban debidamente registrados en la base de datos de la Secretaría Regional Ministerial de Educación y finalmente los cursos. Se incluyeron en este estudio todos los escolares de 6 a 12 años que asistían a los establecimientos educacionales seleccionados y aceptaron participar en el estudio, previa autorización y firma del consentimiento informado de los padres y apoderados, además de los asentimientos en los casos que correspondiera, hasta alcanzar el tamaño y representatividad de la muestra estimados. Luego de realizado el examen, se envió en documento privado, a cada padre o tutor un informe del estado de salud oral con las necesidades de tratamiento de cada escolar de modo que fueran atendidos en el servicio de salud correspondiente o bien, si así lo deseaban tuvieron la posibilidad de acceder a atención en la clínica de la Facultad de Odontología de la Universidad de Chile.

La investigación y su metodología fueron autorizadas por el Comité de Ética de Facultad de Odontología de la Universidad de Chile (Acta de aprobación $\mathrm{N}^{\circ}$ 9, fecha 20/11/2014).

\section{Variables y recolección de los datos}

Los exámenes clínicos se realizaron en los establecimientos educacionales en una sala dispuesta para ello por dos examinadores previamente calibrados y recalibrados cada 200 exámenes por cada profesional.

En la calibración de la detección de las lesiones de caries, se obtuvieron valores Kappa de Cohen, 0,94 para intraexaminador y 0,94 para interexaminador. Los sujetos fueron posicionados de manera de recibir la máxima iluminación natural, y el examinador como apoyo contaba con una linterna frontal con luz LED (Energizer $\left.{ }^{\circledR}\right)$, espejos bucales $n^{\circ} 5$ y sondas OMS. Se utilizó el material necesario para garantizar la comodidad del examinado y del examinador y también las medidas de bioseguridad necesarias en este tipo de intervenciones. Para el cálculo del índice COPD y el ceod se utilizaron los criterios OMS para la detección de lesiones de caries,dientes perdidos y obturados ${ }^{(15)}$, dado que estos criterios permiten la comparabilidad con otros estudios y presentan una mayor facilidad para la calibración de los examinadores en estudios epidemiológicos. Para caracterizar la distribución de las lesiones de caries en la muestra se utilizó el Índice de Significancia de Caries $\left(\right.$ ISC) ${ }^{(16)}$. Para calcular la necesidad de tratamiento, se utilizó el índice de cuidado $(\mathrm{IC})^{(17,18)}$, también se consideró el valor del subcomponente C (caries) y se aplicó el factor 1,2 que es el rendimiento mínimo de obturaciónes por hora odontológica según las normas del Ministerio de Salud. ${ }^{(19,20)}$

\section{Análisis de los datos}

La información recolectada fue tabulada en una planilla Excel y analizada con el programa estadístico IBM ${ }^{\circledR}$ SPSS 21 para Windows ${ }^{\circledR}$.

Para los valores de prevalencia de caries, se consideró el porcentaje de individuos con índices COPD y ceod $\neq 0$. Se compararon los resultados de COPD/ceod según las distintas variables (sexo, estrato socioeconómico y edad), mediante las pruebas de Kruskall-Wallis y $U$ Mann-Whitney, considerando valor de significancia estadística del 95\% $(p<0,05)$.

\section{RESULTADOS}

Se examinaron 1414 escolares, de los cuales 81 fueron excluidos, 65 por no contar con los primeros molares definitivos completamente erupcionados y 16 portaban aparatos de ortodoncia que impedían realizar el examen de salud oral en forma adecuada, quedando la muestra final de 1333 sujetos, con una edad promedio de 9,03 $\pm 1,24$ años (Tabla1). La prevalencia de caries fue de $24,5 \%$ en dentición permanente (COPD $\neq 0$ ) y de $47,8 \%$ en dentición primaria (ceod $\neq 0$ ).

El índice COPD obtenido fue de $0,48 \pm 1,048$, con valores entre 0 y 9 , el índice ceod $1,67 \pm 2,427$, con mínimo 0 y máximo 13 . El desglose según subcomponentes se muestra en la Tabla 2 y 3 . Al comparar los resultados según el sexo (Tabla 4), los hombres presentaron mayor valor de COPD y ceod que las mujeres $(p<0,05)$. Respecto al estrato socioeconómico se observó que el estrato bajo tiene el valor más alto de COPD, estadísticamente mayor al estrato medio y al alto $(p<0,05)$ mientras que no hubo diferencia entre estos últimos. Similar comportamiento 
Tabla 1: Caracterización de la Muestra

\begin{tabular}{|c|c|c|c|c|c|c|c|c|c|}
\hline & \multicolumn{3}{|c|}{ HOMBRE } & $\begin{array}{c}\text { Total } \\
\text { hombres }\end{array}$ & \multicolumn{3}{|c|}{ MUJER } & $\begin{array}{c}\text { Total } \\
\text { mujeres }\end{array}$ & $\begin{array}{c}\text { TOTAL } \\
\text { MUESTRA }\end{array}$ \\
\hline 6 años & 8 & 45 & 0 & 53 & 14 & 12 & 0 & 26 & 79 \\
\hline 7 años & 16 & 77 & 8 & 101 & 22 & 42 & 20 & 84 & 185 \\
\hline 8 años & 16 & 97 & 28 & 141 & 29 & 82 & 39 & 150 & 291 \\
\hline 10 años & 23 & 52 & 28 & 103 & 31 & 65 & 40 & 136 & 239 \\
\hline 11 años & 23 & 35 & 24 & 82 & 29 & 36 & 33 & 98 & 180 \\
\hline 12 años & 1 & 13 & 15 & 29 & 4 & 14 & 24 & 42 & 71 \\
\hline TOTAL & 113 & 413 & 130 & 656 & 164 & 303 & 210 & 677 & 1333 \\
\hline
\end{tabular}

Tabla 2: Índice COPD y ceod general analizado por subcomponentes (X̄: Promedio; DE: Desviación Estándar ),intervalo de confianza e IC: índice de cuidado

\begin{tabular}{|c|c|c|c|c|c|c|c|}
\hline Subcomponente & $\overline{\mathbf{X}}$ & DE & Mediana & Mínimo & Máximo & \multicolumn{2}{|c|}{ Intervalo de confianza } \\
\hline C & 0,15 & 0,60 & 0 & 0 & 8 & 0,12 & 0,18 \\
\hline 0 & 0,33 & 0,857 & 0 & 0 & 9 & 0,28 & 0,38 \\
\hline $\mathbf{P}$ & 0,01 & 0,116 & 0 & 0 & 2 & 0,00 & 0,02 \\
\hline COPD & 0,48 & 1,048 & 0 & 0 & 9 & 0,42 & 0,54 \\
\hline IC & \multicolumn{7}{|c|}{$68,75 \%$} \\
\hline c & 0,62 & 1,377 & 0 & 0 & 10 & 0,42 & 0,54 \\
\hline 0 & 0,95 & 1,725 & 0 & 0 & 11 & 0,86 & 1,04 \\
\hline e & 0,10 & 0,466 & 0 & 0 & 8 & 0,07 & 0,13 \\
\hline ceod & 1,67 & 2,427 & 0 & 0 & 13 & 1,54 & 1,8 \\
\hline IC & \multicolumn{7}{|c|}{$56,88 \%$} \\
\hline
\end{tabular}

Tabla 3: Índice COPD y ceod de ISC analizado por subcomponentes (X̄: Promedio; DE: Desviación Estándar) intervalo de confianza e IC: índice de cuidado

\begin{tabular}{|c|c|c|c|c|c|c|c|}
\hline Subcomponente & $x^{-}$ & $\mathrm{DE}$ & Mediana & Mínimo & Máximo & \multicolumn{2}{|c|}{ Intervalo de confianza } \\
\hline C & 0,44 & 0,98 & 0 & 0 & 8 & 0,39 & 0,49 \\
\hline 0 & 0,98 & 1,26 & 1 & 0 & 9 & 0,91 & 1,05 \\
\hline $\mathbf{P}$ & 0,03 & 0,20 & 0 & 0 & 2 & 0,02 & 0,04 \\
\hline COPD & 1,45 & 1,38 & 1 & 0 & 9 & 1,38 & 1,52 \\
\hline IC & \multicolumn{7}{|c|}{67,58} \\
\hline c & 1,66 & 1,99 & 1 & 0 & 10 & 1,55 & 1,77 \\
\hline o & 2,58 & 2,17 & 2 & 0 & 11 & 2,46 & 2,70 \\
\hline e & 0,28 & 0,78 & 0 & 0 & 7 & 0,24 & 0,32 \\
\hline ceod & 4,52 & 2,28 & 4 & 2 & 13 & 4,40 & 4,64 \\
\hline IC & \multicolumn{7}{|c|}{57,07} \\
\hline
\end{tabular}

Tabla 4: Promedio de índice COPD/ceod según sexo y estrato socioeconómico. (Las comparaciones son válidas dentro de la misma columna. Los promedios identificados con la misma letra son estadísticamente similares [prueba de Mann-Whitney, $p \geq 0,05]$ ).

\begin{tabular}{|c|c|c|}
\hline Sexo & COPD & ceod \\
\hline Hombre & $0,52 \mathrm{~A}$ & $1,37 \mathrm{~A}$ \\
\hline Mujer & $0,44 \mathrm{~B}$ & $1,97 \mathrm{~B}$ \\
\hline Estrato socioeconómico & COPD & ceod \\
\hline Alto & $0,31 \mathrm{~A}$ & $1,35 \mathrm{~A}$ \\
\hline Medio & $0,44 \mathrm{~A}$ & $1,57 \mathrm{~B}$ \\
\hline Bajo & $0,71 \mathrm{~B}$ & $1,75 \mathrm{~B}$ \\
\hline
\end{tabular}


Tabla 5: Valor promedio de índices COPD y ceod según edad y comparación según edad ( ${ }^{*}$ estadísticamente significativo, valor $p<0,05$ )

\begin{tabular}{c|c|c|c|c|c|c|c}
\hline \multicolumn{2}{c}{} & \multicolumn{5}{c}{ Valor p (Prueba de Mann Whitney) } \\
\hline Edad & & 7 & 8 & 9 & 10 & 11 & 12 \\
\hline & COPD & & & & & & \\
\hline 6 & $\mathbf{0 , 0 1}$ & $0,015^{*}$ & $0,000^{*}$ & $0,000^{*}$ & $0,000^{*}$ & $0,000^{*}$ & $0,000^{*}$ \\
\hline 7 & $\mathbf{0 , 1 2}$ & & $0,036^{*}$ & $0,000^{*}$ & $0,000^{*}$ & $0,000^{*}$ & $0,000^{*}$ \\
\hline 8 & $\mathbf{0 , 2 8}$ & & & $0,014^{*}$ & $0,000^{*}$ & $0,000^{*}$ & $0,000^{*}$ \\
\hline 9 & $\mathbf{0 , 4 4}$ & & & & 0,088 & $0,000^{*}$ & $0,000^{*}$ \\
\hline 10 & $\mathbf{0 , 5 7}$ & & & & & $0,020^{*}$ & $0,000^{*}$ \\
\hline 11 & $\mathbf{0 , 8 4}$ & & & & & & $0,001^{*}$ \\
\hline 12 & $\mathbf{1 , 7 2}$ & & & & & & \\
\hline & $\mathbf{C e o d}$ & & & & & & \\
\hline 6 & $\mathbf{2 , 6 1}$ & 0,68 & 0,782 & 0,664 & $0,001^{*}$ & $0,000^{*}$ & $0,000^{*}$ \\
\hline 7 & $\mathbf{2 , 5 4}$ & & 0,708 & 0,144 & $0,000^{*}$ & $0,000^{*}$ & $0,000^{*}$ \\
\hline 8 & $\mathbf{2 , 2 2}$ & & & 0,156 & $0,000^{*}$ & $0,000^{*}$ & $0,000^{*}$ \\
\hline 9 & $\mathbf{1 , 9 4}$ & & & & $0,000^{*}$ & $0,000^{*}$ & $0,000^{*}$ \\
\hline 10 & $\mathbf{0 , 9 2}$ & & & & & $0,001^{*}$ & $0,000^{*}$ \\
\hline 11 & $\mathbf{0 , 5 5}$ & & & & & & 0,161 \\
\hline 12 & $\mathbf{0 , 3 4}$ & & & & & & \\
\hline
\end{tabular}

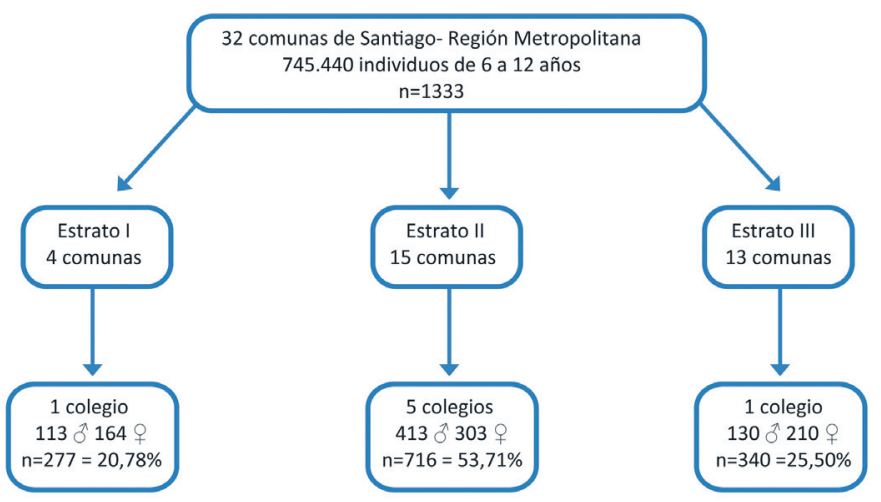

Diagrama 1: Construcción de la muestra representativa multietápica, estratificada y aleatoria

se observó en el índice ceod, donde el estrato alto presentó el menor valor. Los estratos medio y bajo no presentaron diferencias entre sus resultados (Tabla 4).

Al comparar los resultados de los índices por edad, se observó que a medida que aumenta la edad, aumenta el COPD de manera significativa (Tabla 5). Contrariamente, el índice ceod disminuye a medida que aumenta la edad (Tabla 5). Estas diferencias estadísticas se pueden observar en cada año de la muestra mediante la prueba de Mann Whitney (Tabla 5).

La necesidad de tratamiento de la población en estudio es de 167 horas odontológicas para tratar el promedio de 0,15 lesiones de caries encontradas en dentición definitiva, y 689 horas odontológicas para tratar el promedio de 0,62 lesiones de caries en dentición primaria, con un total de 856 horas odontológicas.

\section{Índice de Significancia de Caries (ISC):}

Los valores promedio de COPD y ceod del tercio de la población con mayor índice fueron 1,45 , y 4,52 respectivamente. Aumentando comparativamente los valores de la media de la población que fueron 0,48 y 1,67 respectivamente. Los valores COPD en este grupo aumentan conforme aumenta la edad, mientras el ceod disminuye al aumentar la edad siguiendo la tendencia general de la muestra.

Los valores de los desagregados para COPD y ceod se pueden observar en la tabla 3, donde en este grupo, todos subcomponentes muestran incrementos en relación a la muestra total.

Índice de Cuidado (IC):
El Índice de Cuidados (IC): se define como el número de dientes restaurados como una fracción $(F)$ del número total de dientes cariados $(C)$, perdidos $(P)$ y obturados $(O)$ y nos proporciona informacion valiosa sobre los patrones de atención en los subgrupos por lo tanto, desempeñan un papel útil para resaltar las disparidades en la salud oral. ${ }^{(17)}$

Los IC obtenidos en esta muestra para COPD fue $68,75 \%$, para ceod $56,88 \%$ y en el caso del tercio más afectado de esta población fue $67,58 \%$ para COPD y para ceod $57,07 \%$

\section{DISCUSIÓN}

El presente estudio fue realizado en una muestra representativa de escolares de 6 a 12 años de la Provincia de Santiago, Región Metropolitana y proporciona datos actualizados de prevalencia, y severidad de lesiones de caries estableciendo la necesidad de tratamiento restaurador en este grupo etáreo de escolares chilenos.

La prevalencia de caries encontrada para dentición primaria fue $47,8 \%$, y para dentición permanente fue de $24,5 \%$, con índices ceod 1,67 y COPD 0,48 respectivamente; mientras que el $30 \%$ de la población que presentó mayor severidad de daño correspondió a un índice ISC para ceod 4,52 y para COPD 1,45. Publicaciones previas de prevalencia de caries en escolares tanto nacionales como internacionales, muestran valores diferentes a los encontrados en este estudio. En Chile el trabajo de Cereceda y cols del año 2010 determinó una prevalencia de caries dental de $79,5 \%{ }^{(21)}$, en tanto Villalobos-Rodelo determinó una prevalencia de $82 \%$ en México el año 2006 y Kim de 57,3\% en Korea el año 2017(22,23). Estas diferencias pueden explicarse por los grupos estudiados, ya que el trabajo de Cereceda incluyó una muestra de pacientes de estratos socioeconómicos medio y bajo lo que se relaciona con índices mayores de enfermedad. Respecto a los estudios de Kim y Villalobos-Rodelo, los grupos de estudio incluyeron individuos de hasta 16 años e incluía población rural, lo que podría ser la explicación a la mayor incidencia de caries dental, debido a la relación proporcional de aumento de esta enfermedad con la edad y la ruralidad $(24,25,26)$.

Al extrapolar los valores de COPD/ceod obtenidos con los rangos propuestos por la Organización Mundial de la Salud, se consideran valores "muy bajos" y "bajos" respectivamente ${ }^{(27,28)}$. El diagnóstico de salud oral de preescolares y escolares de Chile realizado por el MINSAL el año 2007 clasificó el COPD de niños de 6 años $(0,16)$ como "muy bajo" y el ceod $(3,71)$ como "moderado". En niños de 12 años el COPD fue clasificado como "bajo"(1,9). En el mismo sentido, el índice de significancia de caries de la población estudiada es menor a la meta propuesta para el año 2015 (COPD 3 máximo en la población de 12 años) ${ }^{(29)}$. Estas diferencias permiten dar cuenta de la reducción alcanzada en estos índices en la última década. Las políticas públicas instauradas en el país, tanto en el sector salud como educacional, apuntan al ámbito promocional y preventivo, entregando herramientas a temprana edad para el autocuidado y fomento de la promoción y prevención de la salud oral. A su vez, cabe mencionar el reforzamiento del componente de Salud Oral en el Modelo de Atención Integral. Es destacable también, el aumento en el subcomponente "O/o", asociándose a un mejor acceso a la salud oral de esta población, considerada como grupo objetivo para el alcance de las metas sanitarias propuestas ${ }^{(30)}$. Sin embargo, es preciso destacar que los índices estudiados corresponden al daño observado clínicamente y que se utilizan en salud pública, debido a que permiten una fácil comparación epidemiológica entre distintas poblaciones, permitiendo comparar con lo previamente publicado, pero no necesariamente reflejan todo el daño de la salud oral existente en los individuos, ya que el examen clínico debería complementarse con otros exámenes, como es el caso de las radiografías que reportaría un $44 \%$ más de lesiones ${ }^{(31,32)}$ y otros métodos visuales directos de diagnóstico que den cuenta de caries incipientes y no cavitadas, como es el Sistema Internacional de Detección y Diagnóstico de Caries, ICDAS ${ }^{(33)}$ ya que ellas, igualmente necesitan ser tratadas y monitorizadas para lograr un mayor control de la enfermedad evitando que estas lesiones iniciales lleguen a cavitarse.

En relación con las diferencias encontradas entre distintos estratos socioeconómicos, Schwendicke y cols, establecieron que en el estrato socioeconómico bajo existe una asociación significativa con un mayor riesgo de tener lesiones de caries o alguna experiencia de caries y esta asociación podría ser más fuerte si se observa en un país desarrollado, debido a que puede encontrarse mayor desigualdad de ingresos en la misma población de estudio ${ }^{(34)}$. Asimismo, Lagerweji y cols concluyeron que en grupos de estratos socioeconómicos altos el número de lesiones de caries en niños de 12 años se reduce en $80-90 \%{ }^{(35)}$. Estas diferencias están asociadas a determinantes sociales como son: nivel educacional, ingreso económico, condición de vivienda, empleo, saneamiento ambiental y acceso a los servicios de salud pero recientemente se ha abierto una nueva arista y una explicación para la diferencia en prevalencia podría 
estar en la influencia de factores epigenéticos, un término que intenta explicar la mayor aparición y severidad de una enfermedad en algunos grupos y que corresponden a alteraciones debido a factores ambientales capaces de modificar la expresión de los genes de un individuo sin alterar la secuencia del DNA, determinando finalmente el padecimiento de cierta condición o la susceptibilidad a desarrollarla ${ }^{(6,36)}$. Aunque los estudios de la influencia de epigenética en odontología aún se encuentran en las primeras etapas, cada vez hay más pruebas de la asociación entre los cambios epigenéticos y las enfermedades periodontales, la respuesta inflamatoria pulpar y el cáncer oral, por ello también, podrían a futuro relacionarse con la caries. Es así que estos factores externos podrían actuar en las últimas etapas de la diferenciación de los osteoblastos dando como resultado una alteración en la mineralización de las estructuras dentales como el esmalte y la dentina, lo que finalmente podría influir en las características de los dientes, la suceptibilidad a desarrollar lesiones de caries y la presentación clínica de ellas. . $^{(7,8,9,36)}$.

Al ser la caries una enfermedad que produce un daño irreversible y acumulativo en el tiempo, la edad es un factor que afecta los resultados de este estudio. Se puede apreciar que a medida que aumenta la edad, el COPD también lo hace, al sumarse las lesiones antiguas con las nuevas, evidenciándose un problema cada vez mayor a medida que avanza la edad $^{(24,25,37)}$. Por el contrario, el índice ceod muestra que a medida que aumenta la edad, va en disminución, ello se explica por la exfoliación y recambio de los dientes primarios cariados y obturados, la cual comienza alrededor de los 6 años de edad hasta los 12-13 años aproximadamente.

Al realizar la comparación de índices COPD/ceod según sexo se observó que en dentición permanente existe mayor prevalencia de caries en mujeres, mientras que el ceod es mayor en hombres. Lukacs y cols, en un meta-análisis del año 2011, mostraron como resultado una cifra de ceod igual o mayor en el grupo de hombres en comparación al de las mujeres, que a medida que avanza la edad se invierte y se mantiene en el tiempo hasta llegar a la adultez, es decir, el índice COPD es mayor en el grupo de mujeres ${ }^{(38)}$. Una explicación podría ser la ubicación de la mujer en un nivel de desventaja al hablar de salud oral, siendo asociado a condiciones genéticas, hormonales, culturales, incluyendo el comportamiento y la heterogeneidad de la dieta. Las diferencias sociales y culturales entre hombres y mujeres podrían influir de distintas maneras el nivel de su salud oral, basado en marcadas diferencias de su vida cotidiana que afectan su exposición tanto a factores de riesgo como protectores ${ }^{(39,40)}$. Por último, las mujeres no solamente serían más propensas a desarrollar lesiones de caries, sino que también acuden más al dentista, aumentando el número de tratamientos realizados, y por ello se observa un incrementado el índice COPD en este grupo basado en el componente O como se ve en un estudio de Shaffer y cols. ${ }^{(41)}$.

En relación con la necesidad de tratamiento restaurador, los resultados obtenidos indican que existe una gran diferencia entre dientes primarios y permanentes, donde los dientes primarios corresponden al $75 \%$ de la necesidad de tratamiento restaurador total. Esto podría explicarse a que por la edad al momento de su evaluación, la dentición primaria había tenido un mayor periodo exposición en la boca siendo afectadas durante más tiempo a agresiones y distintos factores de riesgo determinantes de la aparición de lesiones de caries ${ }^{(25,42,43)}$. Así, la estimación de recursos humanos y tiempo para la realización de tratamiento restaurador en los 745.440 escolares de 6 a 12 años de la Provincia de Santiago, se necesitarán 478.324 horas odontológicas, de las cuales 93.180 son para dientes definitivos y 385.144 en dientes primarios. Esto es dedicar el trabajo de 210 odontólogos y asistentes con 44 horas semanales por todo un año.
Aunque estos valores de prevalencia e ISC son bajos, si se aplica como criterio IC tenemos que el $68,75 \%$ de lesiones ha tenido acceso a la atención en caso de dentición permanente y $56,88 \%$ para dentición primaria, sin embargo aún existe una alta proporción de individuos que necesitan atención tanto en dentición permanente como primaria, y se necesita una gran cantidad de horas profesionales para solucionar el problema de las lesiones de caries presentes. A pesar que con los resultados obtenidos se cumplen los objetivos sugeridos por la FDI y la OMS, para el año 2020 tanto en prevalencia como en Índice de Significancia de Caries, ${ }^{(44,45)}$ se hace perentorio y se requieren mayores esfuerzos para seguir realizando programas de prevención, promoción y educación en salud oral de manera que en un futuro cercano se logre disminuir el impacto de estas enfermedades en la población infantil.

\section{CONCLUSIONES}

A la luz de los resultados obtenidos en este estudio, se puede afirmar que la prevalencia de caries y la severidad de ella en escolares de 6 a 12 años de la Provincia de Santiago han disminuido en relación a los reportados hace una década. Estos índices son mayores en la población socioeconómicamente más vulnerable. Las horas profesionales para cubrir la necesidad de tratamiento restaurador son 856 de las cuales 167 horas son para dentición permanente y 689 horas para dentición primaria.

\section{RESPONSABILIDADES ÉTICAS, PROTECCIÓN DE PERSONAS Y ANIMALES:}

Los autores declaran que los procedimientos seguidos se ajustan siguiendo las normas éticas del comité de experimentación humana responsable y de acuerdo con la Asociación Médica Mundial y la Declaración de Helsinki.

\section{CONFIDENCIALIDAD DE LOS DATOS}

Los autores declaran que se han seguido los protocolos de su centro de trabajo sobre la publicación de datos de pacientes. Derecho a la privacidad y consentimiento informado.

Los autores han obtenido el consentimiento y asentimiento informado de los pacientes, tutores y/o sujetos referidos en el artículo. Estos documentos están en poder del autor de correspondencia.

\section{FINANCIAMIENTO:}

CONICYT, a través del proyecto FONIS SA14ID0056 «Prevalencia de la hipomineralización incisivo-molar en niños de 6 a 12 años y determinación de sus consecuencias clínicas».

\section{CONFLICTO DE INTERESES:}

Los autores declaran no tener conflicto de intereses.

\section{AGRADECIMIENTOS:}

A los doctores/as: Camila Corral quien ayudó en la realización de los exámenes clínicos y a Carlos Canales, Braulio Catalán, Alejandra Contreras e Ignacia Sarquis, quienes ayudaron en la recolección y transcripción de los datos. A CONICYT por el financiamiento a través del proyecto FONIS SA14ID0056.

\section{Bibliografía}

1. Twetman S. Prevention of dental caries as a non-communicable disease Eur J Oral Sci. 2018;126(Suppl. 1):19-25.

2. The challenge of oral disease, a call for global action. 2 edition. (consultado 20 marzo 2018). Disponible en: https://www.fdiworlddental.org.

3. Petersson $\mathrm{GH}$ and Bratthall D. The caries decline: a review of reviews. Eur J Oral Sci. 1996:104(4(Pt 2)):436-443.

4. Urzua I, Mendoza C, Arteaga O, Rodríguez G, Cabello R, Faleiros S, et al. Dental caries prevalence and tooth loss in Chilean adult population: first national denta examination survey. Int J Dent. 2012;2012:810170.

5. Hoffmann A, Spengler D. DNA memories of early social life. Neuroscience. 2014;264:64-75.

6. Mohsin A, Barshaik S. Epigenetics in dentistry: a literature review. J Clin Epigenet. 2017;3:1

7. Fernando S, Speicher DJ, Bakr MM, Benton MC, Lea RA, et al. Protocol for assessing maternal, environmental and epigenetic risk factors for dental caries in children . BMC Oral Health. 2015;15:167.
8. Seo J, Park YJ, Yi YA, Hwang JY, Lee IB, Cho BH et al. Epigenetics: general characteristics and implications for oral health. Restor Dent Endod. 2015;40(1):1422.

9. Packyanathan JS, Juneius CE. Role of epigenetic mechanisms in oral health: a review. Asian J Pharm. 2016;10(Supl.):s472-s479.

10. Fernandes IB, PereiraTS, Souza DS, Ramos-Jorge J, Marques LS, RamosJorge ML. Severity of dental caries and quality of life for toddlers and their families. Pediatr Dent. 2014;39(2):118-123.

11. Sheiham A. Oral health, general health and quality of life. Bull World Health Organ. 2005;83(9):644.

12. Watt R. Emerging theories into the social determinants of health: implications for oral health promotion. Community Dent Oral Epidemiol. 2002;30(4):241-247.

13. Soto L, Jara G y col. Diagnóstico en salud bucal de los niños de 2 y 4 años de edad que asisten a la educación preescolar en la zona norte y centro del país. MINSAL Chile, 2009. 
14. Soto L, Tapia R y col. Diagnóstico nacional de salud bucal del adolescente de 12 años y evaluación del grado de cumplimiento de los objetivos sanitarios de salud bucal 2000-2010. MINSAL, Chile, 2007.

15. World Health Organization. Oral health surveys basic methods. 5th ed. Geneva: WHO; 2013.

16. Bratthall D. Introducing the significant caries index together with a proposal for a new global oral health goal for 12-year-olds. Int Dent J. 2000;50(6):378-84. 17. Gupta E, Inne N, Schuller AA, Vermaire JH, Marshman Z. A scoping review of the uses of the care index in children. Community Dental Health. 2017:34:131-136. 18. Van Dommelen $P$, Schuller AA. The amount of care delivered: challenges of indices in oral health studies. Community Dent Oral Epidemiol. 2016;44(5):485-92. 19. Orientaciones para la programación en red del año 2018. (consultada 20 marzo 2018). Disponible en: www.minsal.cl.

20. Urzúa I. Prevalencia de caries dental y de pérdida de dientes en la población de 65 a 74 años de edad de nivel socio-económico bajo y medio-bajo de la Provincia de Santiago, Región Metropolitana y determinación de los recursos humanos necesarios para su tratamiento. Tesis para optar al grado de Magíster en Ciencias Odontológicas con mención en Cariología. Universidad de Chile, 2009.

21. Cereceda MA, Faleiros S, Ormeño A, Pinto M, Tapia R, Díaz C, et al. Prevalencia de caries en alumnos de educación básica y su asociación con el estado nutricional. Rev Chil Pediatr. 2010;81(1):28-36.

22. Villalobos-Rodelo J, Medina-Solís C, Molina-Frechero N, Vallejos-Sánchez A, Pontigo-Loyola A, Espinoza-Beltrán J. Caries dental en escolares de 6 a 12 años de edad en Navolato, Sinaloa, México: experiencia, prevalencia, gravedad y necesidades de tratamiento. Biomédica. 2006;26:224-33.

23. Kim A, Shim Y, Kim J, An S. Caries prevalence in Korean children and adolescents from 2000 to 2012. J Clin Ped Dent. 2017;41(1):32-37.

24. Zaror C, Pineda P, Orellana J. Prevalencia de caries temprana de la infancia y sus factores asociados en niños Chilenos de 2 y 4 Años. Int $\mathrm{J}$ Odontostomatol. 2011;5(2):171-177.

25. Sarumathi T, Saravana KB, Manjula D, Hemalatha VT, Aarthi N. Prevalence, severity and associated factors of dental caries in 3-6 year old children. J Clin Diagn Res. 2013;7(8):1789-1792.

26. Cabrera C, Arancet MA, Martínez D, Cueto A, Espinoza S. Salud oral en población escolar urbana y rural. Int J Odontostomatol. 2015;9(3):341-348.

27. Vargas V, Krause C, Díaz VP. Estimación del valor promedio del indicador COPD. Chile, 2012. Rev Salud UNINORTE. 2015;31(2):276-283.

28. Vargas-Sanhueza V, Muñoz CK, Díaz-Narváez VP, Molina JF. Oral health status in adult population in Chile (decayed, missing, filled and permanent tooth): clinical observation at national level. Health. 2015;7:1757-1762.

29. Bratthall D, Sweden M. Introducing the significant caries index together with a proposal for a new global oral health goal for 12-year-olds. Int Dent J. 2015;50:378-384.
30. Biblioteca MINSAL. Estrategia nacional de salud / Metas 2011-2020. (consultado el 20 marzo 2018). Disponible en: www.bibliotecaminsal.cl/estrategia-nacional-desalud-metas-2011-2020/

31. Becker T, Levin L, Shochat T, Einy S. How much does the DMFT index underestimate the need for restorative care? J Dent Educ. 2007;71(5):677-681.

32. Labraña G, Pinares J. Diagnóstico radiográfico de la caries dental proximal, una nueva perspectiva. Anuario. Sociedad de Radiología Oral y Máxilofacial de Chile. 2007;10(1):56-63.

33. Shivakumar KM, Prasad S, Chandu GN. International caries detection and assessment system: a new paradigm in detection of dental caries. J Conser Dent. 2009;12(1):10-16.

34. Schwendicke F, Dörfer CE, Schlattmann P, Foster Page L, Thomson WM, Paris S. Socioeconomic inequality and caries: a systematic review and meta-analysis. J Dent Res. 2015;94(1):10-18.

35. Lagerweij MD, Van Loveren C. Declining caries trends: are we satisfied? Curr Oral Health Rep. 2015;2(4):212-217.

36. Gutiérrez S, García D, Santacoloma S, Mejía JP. Dental caries: Do genetics and/or epigenetics influence its etiology? Literature review. Univ Odontol. 2013;32(69):83-92

37. Collins J, Bobadilla M, Fresno MC. Indicadores de riesgo cariogénico en adolescentes de Santo Domingo, República Dominicana. Rev Clin Periodoncia Implantol Rehabil Oral. 2008;1(3):86-89.

38. Lukacs J. Gender differences in oral health in South Asia: Metadata imply multifactorial biological and cultural causes. Am J Hum Biol. 2011;23:398-411.

39. Ferraro M, Viera AR. Explaining gender differences in caries: a multifactorial approach to a multifactorial disease. Int J Dent. 2010: 649643.

40. Martínez-Mier EA, Zandona AF. The impact of gender on caries prevalence and risk assessment. Dent Clin North Am. 2013;57(2):301-315

41. Shaffer J, Lesli E, Feingold E, Govil M, McNeil D, Crout R, et al. Caries experience differs between females and males across age groups in Northern Appalachia. Inter J Dent. 2015;2015:938213.

42. Hara A, Zero D. The Caries Environment: Saliva, Pellicle, Diet, and Hard Tissue Ultrastructure. Dent Clin North Am. 2010;54:455-467.

43. González-Cabezas C. The chemistry of caries: remineralization and demineralization events with direct clinical relevance. Dent Clin North Am. 2010;54:469-478.

44. Global goals for oral health in the year 2000. FDI. Int Dent J. 1982;32:74-77.

45. Hobdell M, Petersen PE, Clarkson J, Johnson N. Global goals for oral health 2020. Int Dent J. 2003;53:285-288. 